\title{
Appendicitis Inflammatory Response Score in Comparison to Alvarado Score in Acute Appendicitis
}

\author{
Toney Jose, MS, DNB ${ }^{1}$ PS Rajesh, MS² \\ ${ }^{1}$ Department of Surgical Gastroenterology, Bangalore Medical \\ College and Research Institute, Bangalore, India \\ 2 Department of General Surgery, Government Medical College, \\ Kottayam, Kerala, India
}

Surg J (NY) 2021;7:e127-e131

\begin{abstract}
Address for correspondence Toney Jose, MS, DNB, Department of Surgical Gastroenterology, Bangalore Medical College and Research Institute, Bangalore 560018, India (e-mail: toneyonly@gmail.com).
\end{abstract}

\begin{abstract}
Keywords

- appendicitis

- clinical prediction rule

- Alvarado

- appendicitis inflammatory response score

Appendicitis is a common differential diagnosis of right lower quadrant pain. Clinical evaluation alone results in high negative appendicectomy rates. Alvarado scoring is the most commonly used clinical prediction rule. The study aimed to compare the recently developed appendicitis inflammatory response (AIR) score with the Alvarado score. This cross-sectional observational study included patients who underwent appendicectomy for clinical suspicion of appendicitis. The clinical and laboratory parameters required for obtaining Alvarado score and AIRS were gathered. Area under ROC curve was calculated for both Alvarado score and AIRS. The study included 130 patients (77 males and 53 females). The negative appendicectomy rate was $10.7 \%$. The perforation rate was $10.3 \%$. The area under ROC for Alvarado score was 0.821 and for AIR score was 0.901 . The Alvarado score had a sensitivity of $72 \%$ and a specificity of $79 \%$ at score $\geq 6$. The appendicitis inflammatory response score had a sensitivity of $98 \%$ for scores $\geq 5$ and a specificity of $97 \%$ for score $\geq 6$. The C-reactive protein (CRP) value was the best performing individual parameter with an area under ROC of 0.789 , followed by WBC count with an area under ROC of 0.762 . Appendicitis inflammatory response score is a recently developed score that outperforms the Alvarado score. AIR score has a higher specificity. The sound construction, gradation of parameters, the inclusion of CRP, and avoidance of subjective parameters make the AIR score an attractive clinical prediction rule which can decrease the rate of negative appendicectomy.
\end{abstract}

With a lifetime incidence of $7 \%$, acute appendicitis is a common clinical condition, one of the many differential diagnoses of a patient presenting with acute right iliac fossa pain. ${ }^{1}$ Many of the patients presenting to surgical emergency with acute right iliac fossa pain, have alternative pathologies. A negative appendicectomy rate up to $17.5 \%$ is noted in India following appendicectomy based on clinical suspicion. ${ }^{2}$ This necessitates accurate diagnosis prior to performing appendicectomy. Controversies exist regarding the management of

received

April 8, 2021 accepted after revision May 17, 2021
DOI https://doi.org/ $10.1055 / \mathrm{s}-0041-1731446$. ISSN 2378-5128. equivocal cases, with some advocating early surgical intervention and others opting for active observation. ${ }^{3-5}$ Many clinical and imaging tools have been developed to aid in the diagnosis of acute appendicitis. Clinical scoring systems like appendicitis inflammatory response (AIR) score, ${ }^{6}$ Alvarado, 7 RIPASA, pediatric appendicitis score, etc, with varying sensitivity and specificity. Imaging modalities like ultrasonography and computer tomography are also used to evaluate for inflamed appendix. ${ }^{8}$ 
Even though the Alvarado score is the most extensively evaluated in validation studies, it has some drawbacks. ${ }^{9,10}$ It was developed by retrospective analysis of patients who underwent appendicectomy for suspected appendicitis and the dichotomization of variables has led to decreased discriminative ability. AIR score was constructed from a prospective cohort, with the grading of variables for greater discrimination, lesser reliance on symptoms, and more on clinical signs. ${ }^{7}$ It also includes C-reactive protein (CRP) as a component with graded scoring. CRP is found to be well correlated with acute appendicitis in many studies. ${ }^{11,12}$ AIR score assigned patients to a high probability zone with substantially higher specificity (97\%) and positive predictive value (88\%) than the Alvarado score (76 and $65 \%$, respectively). ${ }^{13}$

The use of scoring systems is imperative in a resourcelimited setting like India. It is useful in the clinical diagnosis, early referral from primary health care facilities, and for deciding on surgical intervention especially when imaging is inconclusive or not available. The study objective was to compare the performance of appendicitis inflammatory response score and Alvarado score among patients who underwent appendicectomy for suspected acute appendicitis.

\section{Methods}

This cross-sectional observational study was performed in the Department of General Surgery of Government Medical College, Kottayam from January 2017 to June 2018 after obtaining ethical clearance from the Institutional Review Board. Based on sample size calculation the minimum sample size was estimated to be 122 . Consecutive patients aged 12 to 60 years, who were referred to the General Surgical team on-call with acute right iliac fossa pain and were operated on for suspected acute appendicitis during the study period and gave consent for the study were included. Patients without documented signs, symptoms, and biochemical parameters required for calculating AIR score and Alvarado score, those without histopathology report of the resected specimen, and those with chronic abdominal pain were excluded from the study. The decision to operate was taken by a senior surgical staff member. Variables that were recorded included presence of nausea, vomiting, anorexia, migration of pain to the right lower quadrant (RLQ), pain in the RLQ tenderness in RLQ, rebound tenderness or muscular defense, body temperature, white blood cell (WBC) count, proportion of polymorphonuclear leukocytes, and CRP. Alvarado score included migration of pain (score-1), anorexia (score-1), nausea or vomiting (score-1), tenderness in RLQ (score-2), rebound pain (score-1), elevated temperature (score-1), leukocytosis (score-2), and shift to left (score-1). ${ }^{7}$ AIR score included vomiting (score-1), pain in RLQ (score-1), rebound tenderness or muscular defense (light-score 1; medium-score 2; strong-score 3), temperature $\left(>38.5^{\circ} \mathrm{C}\right.$-score 1$)$, polymorphonuclear leukocytes (70-80\%: score 1 ; $\geq 85 \%$ : score 2 ), WBC count (10.0$14.9 \times 10^{3} / \mathrm{mcl}$ : score $1 ; \geq 15.0 \times 10^{3} / \mathrm{mcl}$ : score 2$)$, and CRP (1-4.9 mg/L: score $1 ; \geq 5 \mathrm{mg} / \mathrm{L}$ : score 2$){ }^{6}{ }^{6}$ The diagnosis was confirmed by histopathology in all resected specimens.
Appendicitis was pathologically diagnosed when infiltration of the muscularis propria by neutrophil granulocytes was seen. For the purpose of the study, all histologically confirmed cases were considered to have appendicitis. Statistical analysis was performed using SPSS statistical software 23 (SPSS Inc, Chicago, IL). Continuous variables are described as mean $( \pm S D)$. Comparison of continuous variables was done by independent-samples $t$-tests. Diagnostic accuracy was analyzed using receiver operating characteristic (ROC) curves. Statistical significance was attributed at the $5 \%$ level.

\section{Results}

From January 2017 to June 2018, 130 patients ( 77 males and 53 females) who underwent appendicectomy for suspected acute appendicitis and met the inclusion and exclusion criteria were included in the study. The mean age was $27.5 \pm 13.3$ (range 12-70). 14 patients (six females and eight males) had normal appendix in histology giving a negative appendicectomy rate of $10.7 \%$. The mean Alvarado score among patients with acute appendicitis in histology was $7.4 \pm 1.42$ as compared with $5.6 \pm 1.08$ among patients with normal appendix $(p<0.001)$. The AIR scores were $7.3 \pm 2.23$ and $4.21 \pm 0.97(p<0.001)$, respectively. Thirteen patients $(10.3 \%)$ had a perforated appendix at surgery. The mean Alvarado score was 7.25 for nonperforated appendix and 8.46 for perforated appendix ( $p=0.004$ ). The mean AIR score for nonperforated cases was 6.99 and 10.23 for perforated cases $(p<0.001$.)

The area under the ROC curve was 0.901 for the AIR score as compared with 0.821 for the Alvarado score (-Fig. 1). The Alvarado score has a sensitivity of $72 \%$ and a specificity of $79 \%$ at a score $\geq 6$. For score $\geq 7$, the sensitivity dropped to $46 \%$ whereas the specificity reached $93 \%$. The AIR score showed a sensitivity of $98 \%$ for scores $\geq 5$ with a specificity of $36 \%$. The

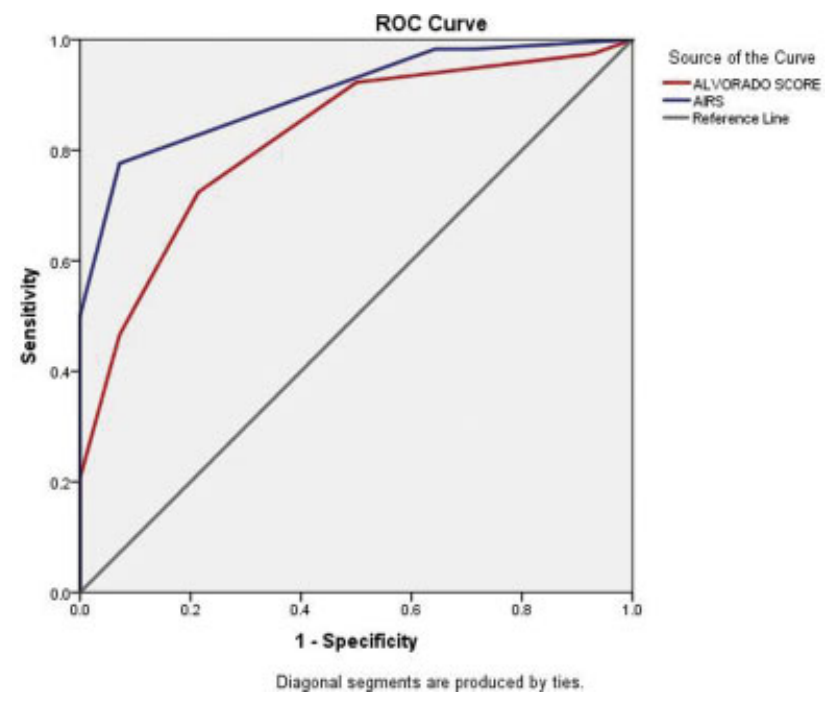

Fig. 1 The combined ROC curves for Alvarado score and AIR score. The area under ROC for AIR score is 0.901 and the area under ROC for Alvarado score is 0.821 . AIR score outperforms Alvarado score in predicting acute appendicitis. AIR, appendicitis inflammatory response; ROC, receiver operating characteristic. 


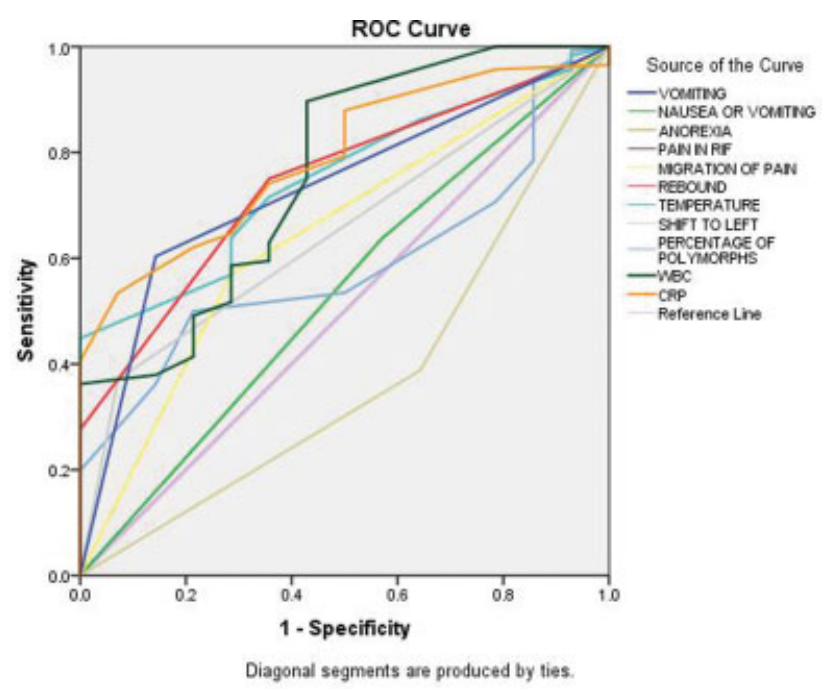

Fig. 2 The ROC curves for individual parameters. The areas under ROC are-vomiting (0.73), nausea or vomiting (0.533), anorexia (0.373), pain in right iliac fossa $(0.50)$, migration of pain (0.642), rebound tenderness $(0.744)$, temperature $(0.75)$, shift to left $(0.650)$, percentage of polymorphs (0.589), white blood cell count (0.762), and C-reactive protein (0.789). The best performing among the individual parameters was the CRP and the worst performing was anorexia. CRP, C-reactive protein; ROC, receiver operating characteristic.

score $\geq 6$ AIR score had a sensitivity of $77 \%$ with a specificity of $97 \%$.

The ROC curves for the individual parameters are shown in - Fig. 2. Vomiting alone has a better area under ROC than nausea and vomiting combined ( 0.73 vs. 0.53 ). CRP value was the best performing with an area under ROC of 0.789 , followed by WBC count with an area under ROC of 0.762 . The poorest performing among symptoms and signs was anorexia with an area under ROC of 0.37 and the poorest performing laboratory investigation was shifted to left with an area under ROC of 0.65 . CRP of $\geq 10.5$ had a sensitivity of $74.1 \%$ and specificity of $64.3 \%$, whereas a CRP $\geq 11.5$ had a sensitivity of $64.7 \%$ and a specificity of $71.4 \%$.

\section{Discussion}

Appendicectomy is often the first major surgery to be undertaken by a surgeon in training. The clinical findings supplemented by investigations help in confirming the clinical suspicion. The use of radiological imaging has resulted in a decrease in the rates of negative appendicectomies. However, the imaging facilities are not accessible to all, especially in rural areas and, also during the night hours-when radiology facilities are not available in many hospitals. Also, imaging can be inconclusive at times. This makes the physician rely on the various clinical prediction scores.

Flum et al analyzed the data from the Washington State Database and identified 63,707 patients who underwent appendicectomy and they have noted a negative appendicectomy rate of $15.5 \%{ }^{14}$ Sharma et al noted a negative appendicectomy rate of $23.72 \%$, which was $13.43 \%$ in males and $37.25 \%$ in females. ${ }^{2}$ Güller et al in an analysis based on the prospective database of the Swiss Association of Laparoscopic and Thoracoscopic Surgery (SALTS) which included patients aged 12 years and over undergoing emergency laparoscopic appendicectomy between 1995 and 2006 noted a negative appendicectomy rate of $6.5 \%$ and a perforation rate of $16.5 \%{ }^{15}$ In our study of 130 patients, the negative appendicectomy rate was noted to be $10.7 \%$. The mean age of the patients who had negative appendicectomy was 19.9. This was in contrast to the mean age of patients with appendicitis in histopathology which was $28.4(p=0.005)$. This indicates the lower threshold in operating on young individuals as compared with the elderly.

AIR score was developed by Andersson and Andersson using the prospective data of 545 patients with suspected acute appendicitis. Comparing the AIR score and Alvarado score, the authors reported an area under ROC of 0.97 versus 0.92 $(p=0.0027)$ for patients with advanced appendicitis and 0.93 versus $0.88(p=0.0007)$ for all patients with appendicitis. Sixty-three percent of the patients were classified into the lowor high-probability group with an accuracy of $97.2 \%$, leaving $37 \%$ in intermediate probability for further investigation. Seventy-three percent of the nonappendicitis patients were correctly classified into low probability. Sixty-seven percent of the advanced appendicitis patients and $37 \%$ of all appendicitis patients were correctly classified into the high-probability zone. ${ }^{6}$ De Castro et al among 941 consecutive patients with suspected acute appendicitis noted that the area under the ROC curve of the AIR score was significantly better than that for the Alvarado score ( 0.96 vs. $0.82, p<0.05$ ). The AIR score also outperformed the Alvarado score in more difficult patients, including women, children, and the elderly. ${ }^{16}$

Kollár et al evaluated the AIR score and compared its performance in predicting risk of appendicitis to both the Alvarado score and the clinical impression of a senior surgeon. The three methods of assessment stratified similar proportions (approximately 40\%) of patients to a low probability of appendicitis $(p=0.233)$. The false-negative rate was $<8 \%$ and did not differ between the AIR score, Alvarado score, or clinical assessment. The Alvarado score had a higher specificity and positive predictive value than the Alvarado score (97 and 88\% vs. 76 and $65 \%$ ) in assigning patients to high probability zone. ${ }^{13}$

Comparison between AIR score, Alvarado score, and Pediatric Appendicitis score was done by Macco et al in a retrospective study on 747 children ( $<18$ years). It was noted that the area under the receiver-operating curve for the AIRS, Alvarado score, and the Pediatric Appendicitis score was $0.90,0.87$, and 0.82 , respectively $(p<0.05)$. In children with a low-risk acute appendicitis, false-negative rates of 14,7 , and $18 \%$ were seen for the AIR score, Alvarado score, and the Pediatric Appendicitis score. ${ }^{17}$ In a randomized trial on AIR score-based management of patients with suspected appendicitis, among low-risk patients, the use of the AIR score-based algorithm resulted in less imaging (19.2 vs. $34.5 \% ; p<0.001$ ), fewer admissions (29.5 vs. $42.8 \% ; p<0.001$ ), fewer negative explorations ( 1.6 vs. $3.2 \% ; p=0.030$ ), and fewer operations for nonperforated appendicitis ( 6.8 vs. $9.7 \% ; p=0.034) .^{18}$

In this study, the area under ROC for Alvarado score was 0.821 and for AIR score was 0.901. The Alvarado score 
showed better sensitivity whereas the AIR score showed better specificity. The Alvarado score has a sensitivity of $72 \%$ and a specificity of $79 \%$ at a score $\geq 6$. When considering score $\geq 7$, the sensitivity dropped to $46 \%$ whereas the specificity reached $93 \%$. The AIR score had a sensitivity of $98 \%$ for scores $\geq 5$ with a specificity of $36 \%$. Score $\geq 6$ showed a sensitivity drop to $77 \%$ with a specificity of $97 \%$. Scott et al noted a high sensitivity (90\%) for AIR score of 5 or more (intermediate and high risk) among all severities of acute appendicitis which increased to $98 \%$ for advanced appendicitis. Ninety-seven percent specificity was noted for a score of 9 or more (high risk) with $70 \%$ among them having perforation or gangrene. ${ }^{19}$

In a meta-analysis by Frountzas et al RIPASA score was found to have an area under the curve of 0.94 as compared with the Alvarado score with $0.79 .^{20}$ Chisthi et al noted that the Modified Alvarado score had an area under the curve of 0.72 as compared with 0.94 for the AIR score and 0.91 for the RIPASA score. ${ }^{21}$ In their study of 107 prospective patients, the AIR score outperformed the modified Alvarado and the RIPASA scoring systems.

In the present study, it was seen that vomiting alone had a better area under ROC than nausea and vomiting combined. This shows the lack of sensitivity of the subjective symptom of nausea and the better discriminating ability of the objective parameter-vomiting. Anorexia-another subjective parameter-also had poor performance. Migratory pain, rebound tenderness, WBC count, and CRP value, all had an area under ROC above 0.7. Considering individual parameters, the CRP value was the best performing with an area under ROC of 0.789 , followed by WBC count with an area under ROC of 0.762 . The poorest performing among symptoms and signs was anorexia with an area under ROC of 0.37 . The poorest performing laboratory investigation was shift to left with an area under ROC of 0.65. CRP was the best overall performing individual parameter in AIRS. WBC count was the best performing individual parameter in the Alvarado score. However, the combined scores of Alvarado and AIR score are better than individual parameters in suspected acute appendicitis.

This study has some limitations. First it is a relatively small size of the study population. Second is the crosssectional nature of the study. Third, limitation is that only patients who underwent appendicectomy for suspected acute appendicitis were included. Fourth is the observational nature of the study. A prospective trial including patients with suspected acute appendicitis, evaluating the treatment decisions based on AIR score is required in the future. Despite these limitations, it is seen from this study and available literature that the AIR score outperforms the Alvarado score as a clinical prediction rule in patients with suspected acute appendicitis with higher specificity. The score is also seen to correlate with the severity of acute appendicitis. Use of AIR score may thus decrease the rate of negative appendicectomies. The AIR score is especially useful in situations where imaging is inconclusive and also in resource-limited areas where radiological imaging is not available.

\section{Conclusion}

The AIR score which is a recently developed score outperforms the Alvarado score. The ease of application of the score, the sound construction, grading of parameters, avoiding of subjective parameters, and the introduction of CRP into scoring all make the appendicitis inflammatory response score into an attractive clinical prediction rule with higher specificity. The use of AIR score in resource-limited settings can decrease the rate of negative appendicectomy.

\section{Ethics Approval}

Approval was obtained from the Ethics Committee of Govt. Medical College, Kottayam. The procedures used in this study adhere to the tenets of the Declaration of Helsinki.

\section{Consent to Participate}

Informed consent was obtained from all individual participants included in the study.

\section{Availability of Data and Material}

Yes.

\section{Authors' Contributions}

T.J. was involved in the study conception and design, data collection and analysis, and manuscript preparation. R.P.S. was involved in the study conception, design, analysis, and manuscript preparation.

\section{Funding}

None.

\section{Conflicts of Interest}

None declared.

\section{Acknowledgment}

None.

\section{References}

1 McCartan DP, Fleming FJ, Grace PA. The management of right iliac fossa pain - is timing everything? Surgeon 2010;8(04):211-217

2 Sharma R, Kasliwal DK, Sharma RG. Evaluation of negative appendicectomy rate in cases of suspected acute appendicitis and to study the usefulness of ultrasonography in improving the diagnostic accuracy. Indian J Surg 2007;69(05):194-197

3 Velanovich V, Satava R. Balancing the normal appendectomy rate with the perforated appendicitis rate: implications for quality assurance. Am Surg 1992;58(04):264-269

4 Andersson R, Hugander A, Thulin A, Nyström PO, Olaison G. Indications for operation in suspected appendicitis and incidence of perforation. BMJ 1994;308(6921):107-110

5 Bachoo P, Mahomed AA, Ninan GK, Youngson GG. Acute appendicitis: the continuing role for active observation. Pediatr Surg Int 2001;17(2-3):125-128

6 AnderssonM,Andersson RE. Theappendicitisinflammatory response score: a tool for the diagnosis of acute appendicitis that outperforms the Alvarado score. World J Surg 2008;32(08):1843-1849

7 Alvarado A. A practical score for the early diagnosis of acute appendicitis. Ann Emerg Med 1986;15(05):557-564 
8 Nautiyal H, Ahmad S, Keshwani NK, Awasthi DN. Combined use of modified Alvarado score and USG in decreasing negative appendicectomy rate. Indian J Surg 2010;72(01):42-48

9 Owen TD, Williams H, Stiff G, Jenkinson LR, Rees BI. Evaluation of the Alvarado score in acute appendicitis. J R Soc Med 1992;85 (02):87-88

10 Douglas CD, Macpherson NE, Davidson PM, Gani JS. Randomised controlled trial of ultrasonography in diagnosis of acute appendicitis, incorporating the Alvarado score. BMJ 2000;321 (7266):919-922

11 Andersson RE. Meta-analysis of the clinical and laboratory diagnosis of appendicitis. Br J Surg 2004;91(01):28-37

12 Ransohoff DF, Feinstein AR. Problems of spectrum and bias in evaluating the efficacy of diagnostic tests. N Engl J Med 1978;299 (17):926-930

13 Kollár D, McCartan DP, Bourke M, Cross KS, Dowdall J. Predicting acute appendicitis? A comparison of the Alvarado score, the Appendicitis Inflammatory Response Score and clinical assessment. World J Surg 2015;39(01):104-109

14 Flum DR, Morris A, Koepsell T, Dellinger EP. Has misdiagnosis of appendicitis decreased over time? A population-based analysis. JAMA 2001;286(14):1748-1753

15 Güller U, Rosella L, McCall J, Brügger LE, Candinas D. Negative appendicectomy and perforation rates in patients undergoing laparoscopic surgery for suspected appendicitis. Br J Surg 2011; 98(04):589-595

16 de Castro SM, Ünlü C, Steller EP, van Wagensveld BA, Vrouenraets BC. Evaluation of the appendicitis inflammatory response score for patients with acute appendicitis. World J Surg 2012;36(07): 1540-1545

17 Macco S, Vrouenraets BC, de Castro SM. Evaluation of scoring systems in predicting acute appendicitis in children. Surgery 2016;160(06):1599-1604

18 Andersson M, Kolodziej B, Andersson RESTRAPPSCORE Study Group. Randomized clinical trial of Appendicitis Inflammatory Response score-based management of patients with suspected appendicitis. Br J Surg 2017;104(11):1451-1461

19 Scott AJ, Mason SE, Arunakirinathan M, Reissis Y, Kinross JM, Smith JJ. Risk stratification by the Appendicitis Inflammatory Response score to guide decision-making in patients with suspected appendicitis. Br J Surg 2015;102(05):563-572

20 Frountzas M, Stergios K, Kopsini D, Schizas D, Kontzoglou K, Toutouzas K. Alvarado or RIPASA score for diagnosis of acute appendicitis? A meta-analysis of randomized trials. Int J Surg 2018;56:307-314

21 Chisthi MM, Surendran A, Narayanan JT. RIPASA and air scoring systems are superior to Alvarado scoring in acute appendicitis: diagnostic accuracy study. Ann Med Surg (Lond) 2020;59:138-142 